East African Medical Journal Vol. 77 No. 7 July 2000

EXPERIENCE WITH IUCD INSERTION OUTSIDE OF MENSES IN KENYA

D.A. Kokonya, MBBS, Research Project Physician, S.K.A. Sinei, MBBS, MMed, Associate Professor, Department of Obstetrics/Gynecology, C.B. Sekadde-Kigondu, PhD., Associate Professor, Department of Clinical Chemistry, College of Health Sciences, University of Nairobi, P.O. Box 19676, Nairobi, Kenya, C.S. Morrison, PhD., Senior Epidemiologist, C. Kwok, MSPH, Epidemiologist and D.H. Weiner, MPH, Biostatistian, Family Health International, Research Triangle Park, NC, 27709. U.S.A

Request for reprints to: Professor C. Sekadde-Kigondu, Department of Clinical Chemistry, College of Health Sciences, University of Nairobi, P.O. Box 19676, Nairobi, Kenya.

\title{
EXPERIENCE WITH IUCD INSERTION OUTSIDE OF MENSES IN KENYA
}

\author{
D.A. KOKONYA, S.K.A. SINEI, C.B. SEKADDE-KIGONDU, C.S. MORRISON, C. KWOK and D.H. WEINER
}

\begin{abstract}
Objective: To determine if women receiving intrauterine devices (IUCDs) outside of menses have an acceptable rate of insertion problems and subsequent IUCD-related complications. Design: Cross-sectional and prospective cohort study of insertions at times other than during menses.

Setting: The study was carried out in two government family planning (FP) clinics in Nairobi, Kenya.

Subjects: After appropriate pre-test and post-test HIV counselling, 1686 women requesting IUCDs at two FP clinics between 1994 and 1995 in Nairobi were enrolled at baseline into a study examining the effect of human immuno-deficiency virus (HIV) infection on IUCDrelated complications. Six hundred and forty nine women (156 HIV-infected and $493 \mathrm{HIV}$ uninfected) were selected for the four month follow up study. They were classified according to their menstrual cycle status at time of IUCD insertion.

Main outcome measures: Problems at the time of insertion (pain, bleeding, immediate expulsion) and IUCD-related complications through four months.

Results: Rates of immediate insertion problems were low in the women who had insertions during menses $(\mathbf{7 . 0 \%})$, outside of menses $(4.0 \%)$ or had oligomenorrhea/amenorrhea $(2.6 \%)$. The adjusted odds ratios for IUCD insertion problems outside of menses and in oligomenorrhea/ amenorrhea (versus women with insertion during menses) were 0.54 (95\% CI 0.18 -1.59) and $0.39(95 \%$ CI $0.12-1.29)$ respectively. IUCD-related complications were higher in the oligomenorrhea/amenorrhea $(\mathbf{1 1 . 5 \%})$ or insertion outside of menses $(6.9 \%)$, than the within menses $(\mathbf{4 . 3 \%})$ groups. However, the differences were not statistically significant. Adjusted odds ratios for IUCD outside of menses and oligomenorrhoea/amenorrhea groups were 1.65 (95\% CI 0.21 - 12.91) and 2.72 (95\% CI 0.34 - 21.71) respectively.

Conclusion: The results confirm that the IUCD can be safely inserted outside of menses with minimal insertion difficulties and subsequent complications. Availability of IUCDs outside of menses may enhance IUCD acceptance in Kenya and create better opportunity for visual screening of the cervix for sexually transmitted infections.
\end{abstract}

\section{INTRODUCTION}

Intrauterine devices (IUCD) are used by approximately 100 million women worldwide and are a highly effective, safe, inexpensive and long-lasting contraceptive $\operatorname{method}(1)$. The Copper $(\mathrm{Cu}) \mathrm{T} 380 \mathrm{~A}$, one of the latest generation copper IUCDs, provides safe and effective contraception $(99 \%)$ for 10 years and has a very low expulsion rate $(1,2)$.

Although their user effectiveness is higher than oral contraceptives and implants, trends over a decade in family planning use in Kenya show that, while IUCD showed only a modest increase in uses, injections have experienced a particularly steep increase (1-7\%), pill use has tripled, and female sterilisation doubled(3). Nevertheless, the relative decline in IUCD use in Kenya has been attributed to five interrelated factors; poor quality of care, fear of HIV acquisition/transmission, poor product image, provider bias or preference and shifting client preference(4).
IUCDs can be inserted safely at any time of the menstrual cycle as long as pregnancy and other contra-indications are ruled out $(1,5)$. With the development of plastic devices in the 1960s, insertion was frequently done during the menstrual period, when the cervix may be more open. The availability of resilient copper IUCDs provided further freedom in the timing of fitting. Although some authors contend that a relationship exists between timing of insertion and rates of expulsion, pregnancy, and removals for pain/bleeding, this relationship is weak and rigid adherence to insertion of an IUCD during menses may be less important than the benefits to be gained by insertion upon request(6). In Kenya, the IUCD insertion for many years has been restricted to the period during menses. This period is advantageous to the providers since pregnancy can be ruled out unequivocally and providers perceive that this timing enhances ease of insertion and minimises menstrual disturbances.

Also bleeding problems can be masked if insertion is done during menses. However, insistence on insertion 
during menses may result in a potential user either becoming pregnant while waiting for the next menses or defaulting in coming back to the clinic.

Use of a simple checklist to rule out pregnancy has been recently tested and proved valid(7). This suggests that IUCD insertion outside of menses can be done with minimal risk of inserting into a pregnant uterus. Moreover, in areas with high rate of sexually transmitted infections (STIs), insertion during menses precludes the opportunity to carefully examine the cervix and vagina for signs of STIs to reduce dissemination of infections at the time of the IUCD insertion. Other advantages of inserting an IUCD outside of menses are easy and more accurate assessment of cervical os combined with accurate uterine sounding to reduce the chance of uterine perforation. Visual assessment of the cervix can also help in screening for malignancy especially if it is combined with the ability for specimen collection such as the Pap smear.

This paper describes the experience of IUCD insertion outside of menses in a large cohort of Kenyan women and makes recommendations for IUCD service delivery guidelines based on these findings.

\section{MATERIALS AND METHODS}

Study population and procedures: Details of study methods have been described elsewhere(8). Briefly, a total of 1702 women were referred to the study. Upon consultation with study staff, five women chose not to have an IUCD inserted and 11 women were examined but did not have an IUCD inserted for medical reasons (including six women with mucopurulent cervical discharge, overt cervicitis or abdominal tenderness). In all, 1686 women attending two family planning clinics in Nairobi, between 1994-1995 requesting an IUCD were recruited into the study. Because of the need to collect cervical specimens for HIV DNA testing outside of menses, menstruating women were asked to return not more than five days after menses according to the last menstrual period (LMP). However, some women returned while bleeding or spotting.

After written informed consent was obtained and HIV pretest counselling conducted, blood was drawn for HIV-l serological testing. Study nurses conducted a brief interview to gather demographic and recent sexual behaviour and contraceptive use information. Physicians conducted a physical examination to assess the condition of the vagina, cervix and any pelvic tenderness and collected an endocervical swab for detection of HIV-1 DNA. The IUCD was inserted if the woman met the local eligibility criteria(9). Additional study eligibility criteria included: no use of antibiotics within 14 days or long-acting penicillin within the previous month; residing or working in Nairobi, having a sufficient address for follow up and willing to return at one and four months for follow up and agreeing to be tested for HIV infection.

Each physician completed a physical examination form by noting the condition of the vaginal and cervical epithelia, colour and quality and amount of vaginal and cervical discharge, any pelvic, cervical motion or adnexal tenderness, and any problems associated with IUCD insertion.

Process of IUCD insertion: In 1069 clients (63.4\%) IUCDs were inserted without the use of a tenaculum(10). This was done at one site to minimise dangers of creating iatrogenic genital sores or wounds which might enhance disease transmission, painrelated anxiety and bleeding of the cervix. At the other clinic $(\mathrm{n}=$ $617 ; 36.6 \%$ ) a tenaculum was used at all times. At both clinics every effort was made to observe the highest standard of asepsis, especially in the handling of the IUCD $(\mathrm{Cu} 380 \mathrm{~A})$ where no-touch technique was used. Women were then scheduled for follow up at one month but were advised to return earlier if a menstrual period was overdue ( $>2$ weeks), an infection was suspected or if they experienced an adverse reaction to their IUCD.

Follow up component: All the 156 women who tested HIV1 positive and 493 randomly selected HIV-uninfected women who presented within a week's time at the same clinic as the infected woman, were selected for the follow up.

At one month post-insertion, interviews and physical examinations were again conducted and endocervical specimens were collected for diagnosis of $C$. trachomatis and $N$. gonorrhoea. At four months similar clinical and laboratory data collection procedures were conducted except cervical specimens were collected only from symptomatic women. Study physicians for physical examination and specimen collection were masked to participants' HIV status throughout the study.

The laboratory methods have been described in detail previously(8). HIV testing was conducted using two commercially available ELISA kits(8). Women were tested for C. trachomatis antigen using Syva Micro Track II EIA (Syva, Belgium). Endocervical specimens for culture of $N$. gonorrhoea were taken using sterile swabs and inoculated directly onto Thayer-Martin Transport medium according to standard protocol and stored at $4^{\circ}$ $\mathrm{C}$ until used. After inoculation, plates were kept in $\mathrm{CO}_{2}$ jars at room temperature until transported to the laboratory where they were incubated for 48 hours at $37^{\circ} \mathrm{C}$ and read as positive or negative.

Comparison groups and outcome measures: Outcome measures for this sub-study were problems at the time of insertion (pain, bleeding, or immediate expulsion) and IUCD-associated complications including: pelvic inflammatory disease (PID) (11), full or partial IUCD expulsion, pregnancy, or IUCD removal because of infection, pain, or bleeding.

Comparisons of insertion problems at baseline are made among the entire group of 1,686 IUCD acceptors. Comparison of short-term complications by menstrual history groups was limited to the 615 women (144 HIV+, 471 HIV-) for whom longitudinal information was available.

The participants were classified into one of three groups according to their last menstrual period date. The groups were women with blood on the swab and had their IUCD inserted within eight days of LMP (3.4\%) referred as "within Menses" group; women not meeting this criterion but whose LMP was less than 35 days before insertion were grouped as "outside menses" (68.7\%), while women whose last LMP occurred 35 days or more but less than six months, were referred to as oligomenorrhoeic. The women whose menses had occurred more than six months earlier were defined as "amenorrhoeic". The oligomenorrhoeic and amenorrhoeic groups were combined to form a third group (27.9\%). The high-risk period for pregnancy was estimated to be between the 9th to 19th day of the cycle with $15.2 \%$ of women falling in this category while the last days in the cycle (20-35 days) were considered as an infertile period (3.5\%), using the instructions for fertility awareness-based methods(1).

Statistical analysis: Baseline descriptive statistics were provided for all women in the cross-sectional $(n=1686)$ and follow-up cohorts $(\mathrm{n}=615)$. Nineteen women could not be classified because of missing menstrual data, thus leaving 1667 in the study group. Logistic regression was used to estimate the odds ratios of experiencing an insertion problem or IUCD-related complication for women with IUCD insertions outsides of menses and those experiencing oligomenorrhoea or amenorrhoea compared to women with insertion during menses. We used adjusted odds ratios derived from multivariate logistic models to estimate the 
risk ratio. We tested as co-founders: demographic, behavioural, and physical assessment variables. We included co-founders in multivariate models if covariate inclusion changed at least one odds ratio for the groups by at least $10 \%$. Models were tested for goodness of fit with the Homer-Lemeshow statistic. SAS, version 6.12 (SAS Institute, Cary, NC, and USA), was used for all analyses. The type I error rate was set at 0.05 , two-tailed and unadjusted for multiple comparison for all analyses.

\section{RESULTS}

Women among baseline (1686 women) and follow up (615 women) cohorts shared similar characteristics. Study participants were mainly parous (two or more live births), living with a partner, aged over 20 years and had at least secondary education (Table 1). Few participants reported more than one sexual partner or use of condom in the previous three months or a sexually transmitted infections in the year before the baseline visit. About $36 \%$ women had previously used IUCDs.

Table 1

Characteristics of women among baseline and follow up cohorts

\begin{tabular}{lll}
\hline Characteristic & $\begin{array}{l}\text { Baseline } \\
(\mathrm{n}=1686)\end{array}$ & $\begin{array}{l}\text { Follow-up } \\
(\mathrm{n}=615)\end{array}$ \\
$\%$ & $\%$ \\
\hline
\end{tabular}

Socio-demographic factors

Age (years)

$$
<20
$$

$20-29$

$30+$

Live births

None

1

$>2$

Living with partner

Education: $\geq$ Secondary

Occupation

None/housewife

Domestic/labourer Other

Ethnicity

Kikuyu

Luo

Luhya

Other

STI history

STI in the last year

Partner with a possible STI in last year: Yes

Don't Know

Sexual/contraceptive behaviour

Number of partners ${ }^{1}$

None/one

Coital frequency ${ }^{1} \geq 3$ times/week

Any condom use ${ }^{1}$

IUCD use

None

$>3$ months ago

$\leq 3$ months ago

Physical assessment data

Abnormal vaginal discharge

Color of discharge

Clear

White/gray

yellow/green

Bloody

Cervical friability

Strawberry cervix

Cervical oedema

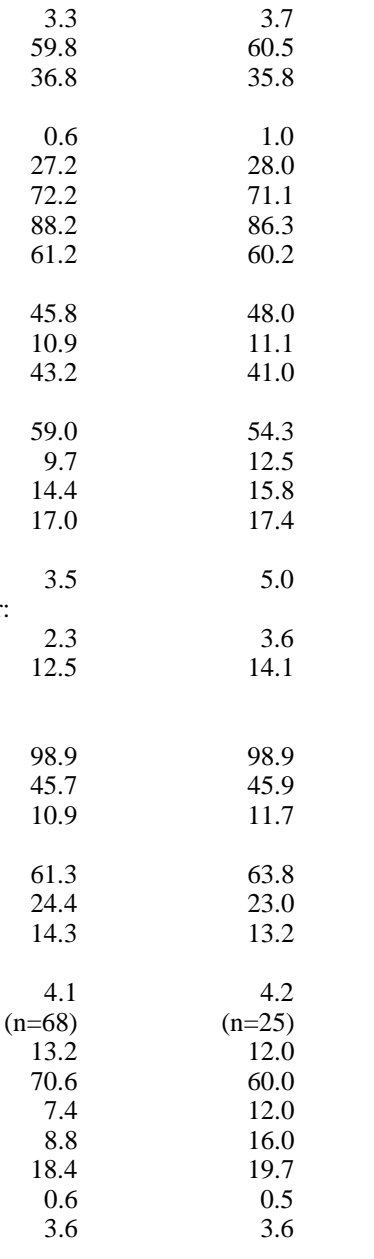

${ }^{1}$ Sexual/contraceptive behavior in 3 months prior to baseline interview
At baseline, about $4 \%$ of the participants had abnormal vaginal discharge. Cervical friability $(<20 \%)$, presence of strawberry cervix $(<1 \%)$ and cervical oedema $(<4 \%)$ were also minimal at baseline.

Proportions of women with insertion problems were low in each of the three menstrual cycle groups including $4.0 \%$ in the outside of menses group, $7.0 \%$ in within menses group and $2.6 \%$ in the oligomenorrhoea/amenorrhoea group (Table 2). Pain was experienced more in the group who had IUCDs inserted outside menses $(2.1 \%)$, than in women whose IUCDs were inserted during menses $(0 \%)$. Immediate expulsions occurred more among the within menses group (7.0\%) than in the outside menses $(2.8 \%)$ and the oligomenorrhoea/amenorrhoea group (1.7\%). This difference was statistically significant $(\mathrm{p}<0.05)$.

An IUCD was inserted in a woman who was later found to be pregnant $(0.06 \%)$. In this case, pregnancy was not ruled out with a laboratory test at recruitment due to a history of prolonged amenorrhoea for two years while having Depo-Provera injections. Pregnancy was confirmed at the one-month visit on examination and a positive laboratory test.

Table 2

Insertion problems by menstrual cycle groups at insertion $(n=1667)^{1,2}$

\begin{tabular}{|c|c|c|c|c|c|c|}
\hline \multirow[t]{2}{*}{ Characteristic } & \multicolumn{2}{|c|}{$\begin{array}{l}\quad(\mathrm{n}=1145) \\
\text { Outside menses }\end{array}$} & \multicolumn{2}{|c|}{$\begin{array}{l}\quad(\mathrm{n}=57) \\
\text { Within menses }\end{array}$} & \multicolumn{2}{|c|}{$\begin{array}{r}\quad(n=465) \\
\text { Oligomennorhoea/ } \\
\text { amenorrhoea }\end{array}$} \\
\hline & No. & $\%$ & No. & $\%$ & No. & $\%$ \\
\hline \multicolumn{7}{|c|}{ Insertion problems } \\
\hline None & 1099 & 96.0 & 53 & 93.0 & 453 & 97.4 \\
\hline Any ${ }^{3}$ & 46 & 4.0 & 4 & 7.0 & 12 & 2.6 \\
\hline Pain & 24 & 2.1 & 0 & 0 & 6 & 1.3 \\
\hline Bleeding & 18 & 1.6 & 1 & 1.8 & 5 & 1.1 \\
\hline $\begin{array}{l}\text { IUCD immedia } \\
\text { expelled }^{4}\end{array}$ & 32 & 2.8 & 4 & 7.0 & 8 & 1.7 \\
\hline
\end{tabular}

1 Nineteen women had missing menstrual information

2 One woman was excluded who received the IUCD while pregnant

3 Number of women with one or more insertion problems

$4 \mathrm{p}=0.05$ for menstrual cycle groups comparison

Crude and adjusted odd ratios for insertion problems according to menstrual grouping at insertion were not statistically significant (Table 3 ). The adjusted odds ratios for IUCD insertion outside of menses and for the oligomenorrhoea/amenorrhoea group were 0.54 (95\% CI 0.18 - 1.59) and 0.39 (95\% CI 0.12 - 1.29) respectively, after controlling for study site, age, education, edema, recent IUCD use and coital frequency. HIV infection was not associated with insertion problems and did not confound the association between menstrual cycle groupings and insertion problems.

We also evaluated whether use of a tenaculum during IUCD insertion was associated with problems immediately following insertion including pain, bleeding or expulsions. There was no difference in either overall problems $(3.2 \%$ 
with a tenaculum; $4.0 \%$ without a tenaculum; $\mathrm{p}=0.42$ ) or in perception of pain $(1.8 \%$ of both groups, $\mathrm{p}=0.99)$ among the two groups.

Table 3

Crude and adjusted odds ratios for insertion problems by menstrual cycle groupings at insertion $(n=1667)$

\begin{tabular}{lll}
\hline & $\begin{array}{l}\text { Crude odds ratio } \\
(95 \% \mathrm{CI})\end{array}$ & $\begin{array}{l}\text { Adjusted odds ratio } \\
(95 \% \mathrm{CI})\end{array}$ \\
\hline $\begin{array}{l}\text { Insertion problems } \\
\text { Within menses group }\end{array}$ & 1.00 & 1.00 \\
$\begin{array}{l}\text { Outside menses group } \\
\text { Oligoamenorrhoea/ }\end{array}$ & $0.56(0.19-1.60)$ & $0.54(0.18-1.59)^{1}$ \\
$\quad$ amenorrhoea & $0.35(0.11-1.13)$ & $0.39(0.12-1.29)^{1}$ \\
\hline
\end{tabular}

${ }^{1}$ Adjusted for study site, age, education, edema, recent IUCD use and coital frequency; Goodness of fit test: $\mathrm{p}=0.80$

There were 49 IUCD-related complications including three cases of PID, 21 expulsions and 24 removals. Failure of method resulted in one pregnancy (Table 4). Proportions of IUCD-related complications were lower in the outside of menses $(6.9 \%)$ and within menses groups $(4.3 \%)$ than oligomenorrhoea/amenorrhoea group (11.5\%). However, the differences were not statistically significant.

Table 4

IUCD-related complications outcome by menstrual cycle groupings at insertion $(N=605) 1$

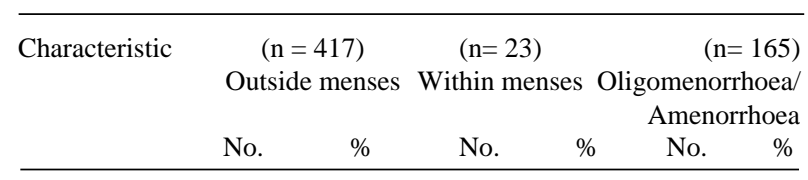

IUCD-related complications

$\begin{array}{lllllll}\text { None } & 388 & 93.1 & 22 & 95.7 & 146 & 88.5\end{array}$

$\begin{array}{lllllll}\text { PID } & 1 & 0.2 & 0 & 0 & 2 & 1.2\end{array}$

$\begin{array}{lrrrrrr}\text { Expulsion } & 10 & 2.4 & 1 & 4.3 & 10 & 6.1\end{array}$

$\begin{array}{lllllll}\text { Removal } & 18 & 4.3 & 0 & 0 & 6 & 3.6\end{array}$

$\begin{array}{lrrrrrr}\text { Pregnancy } & 0 & 0 & 0 & 0 & 1 & 0.6\end{array}$

$1_{10}$ women had missing menstrual information

Table 5

Crude and adjusted odds ratios for IUCD-related complications by menstrual cycle groupings at insertion $(N=605)$

\begin{tabular}{lll}
\hline & $\begin{array}{l}\text { Crude odds ratio } \\
(95 \% \mathrm{CI})\end{array}$ & $\begin{array}{l}\text { Adjusted odds ratio } \\
(95 \% \mathrm{CI})\end{array}$ \\
\hline $\begin{array}{l}\text { IUCD-related complications } \\
\text { Within menses group }\end{array}$ & 1.00 & \\
$\begin{array}{l}\text { Outside menses group } \\
\text { Oligoamenorrhoea/ }\end{array}$ & $1.64(0.21-12.64)$ & $1.65(0.21-12.91) 1$ \\
$\quad$ amenorrhoea & $2.86(0.36-22.47)$ & $2.72(0.34-21.71) 1$ \\
\hline
\end{tabular}

${ }^{1}$ Adjusted for study site, education and used IUCD; Goodness of fit test: $\mathrm{P}=0.5047$

The adjusted odds ratios for IUCD insertion outside of menses and in the oligomenorrhoea/amenorrhoea were
1.65 (95\% CI 0.21 - 12.91) and 2.72 (95\% CI 0.34 - 21.71) respectively after controlling for study site, education, and ever IUCD use (Table 5). HIV infection was not associated with IUCD-related complications and did not confound the association between menstrual cycle groupings and complications.

\section{DISCUSSION}

These results confirm findings from other countries that show that IUCD insertion is safe and effective at any time of the menstrual cycle(12-13). An international study of about 12,000 women who had IUCDs inserted at different times during their menstrual cycles and similar studies in the USA found no advantage over the conventional practice of insertion during the first five days of the menstrual cycle(1). Thus for a woman who is reasonably sure that she is not pregnant, the best time to insert an IUCD is when she comes to a health center to request it (6).

According to United States Agency for International Development's (USAID) Technical Guidance Working Group, in the absence of a laboratory pregnancy test, a provider can be reasonably sure a woman is not pregnant if she has no symptoms or signs of pregnancy. These signs include: no intercourse since last normal menses; correct and consistent use of another reliable contraceptive method within four weeks postpartum for non-lactating women; within seven days post-abortion; fully breast-feeding, amenorrhoeic and less than six months postpartum. These criteria have been validated in Kenya where a study showed an extremely low pregnancy rate of only $1 \%$, in women who came for family planning service outside of menses (7).

The major advantage of inserting an IUCD outside of menses, which has been illustrated in this study, is the ability of the provider to visualise the cervix for abnormal discharge and exclude the client if she has signs of STIs. Severe cervical friability was seen in three patients, again cautioning the provider to revisit the exclusion criteria for these clients. Short-term complications (PID, expulsion, removals and pregnancy) were low in this study. An IUCD was inserted in one woman with amenorrhoea where uncertainty of the ovulatory period is common.

Escape ovulation can unpredictably occur in this group and care should be taken by providers either to exclude pregnancy prior to insertion or ascertain coital frequency and risk of pregnancy in the past months. Insertion of IUCD in the window ovulatory period can lead to unplanned pregnancies.

Immediate expulsion of the IUCDs occurred more in the women who had their IUCDs inserted within menses and the difference was statistically significant $(\mathrm{p}=0.05)$. The expulsion rate during use was observed more in the group with oligomenorrhoea/amenorrhoea. Reasons for removals of IUCDs were due to pain, bleeding and infection with only three cases of PID. The results show that this was a low risk group for reproductive tract infections. The 
presence of blood on the swab signified in the majority of cases menstrual flow, however, in a few cases there was inter-menstrual bleeding. Visual inspection of the vagina and cervix excluded six women judged to have had a cervical infection. A presence of yellow/green discharge in five clients underscores a need for either syndromic management of STIs or where possible a laboratory back up system for testing prior to insertion of IUCDs.

While previous studies suggest that IUCD insertion outside of menses is feasible and safe, few studies have reported on the practical experience of IUCD insertion outside of menses in a large group of Kenyan women. The results of this study indicate the feasibility and practicability of insertion of IUCD outside of menses with minimal complications. The results should motivate health providers to offer IUCDs to women who are not menstruating. This study also concludes that insertion of IUCD outside menses avoids insertion in women with symptomatic cervical infections and the turning away of clients who wish to have an IUCD insertion and are at low risk of pregnancy.

The insertion of IUCD during menses misses those women who have symptomatic STIs that cannot be detected if the client came during menses. Motivational factors such as having a pap smear specimen taken at the same visit and creating an environment suitable for cervical assessment are important health benefits of insertion outside of menses.

The results confirm that the IUCD can be safely inserted outside of menses in Kenya with minimal insertion problems and subsequent IUCD-related complications. Availability of IUCDs outside of menses would enhance IUCD acceptance and create a better opportunity for visual screening of the cervix for STI and malignancies specifically carcinoma of the cervix.

We recommend that health providers in Kenya be updated on the safety of insertion of IUCDs anytime during the menstrual cycle, thus making family planning services more accessible to women especially for those women who come non-menstruating. Stanback et al(14) have estimated that $35 \%$ of women who come for the first time seeking family planning services are sent home due to rigid menstrual requirements. More recently, Stanback et al(7) have validated a simple checklist to rule out pregnancy as a surrogate to an expensive pregnancy test with high negative predictive value of $99 \%$. Counselling regarding IUCD insertion outside menses should also be extended to paramedical staff and those involved in community-based or outreach programmes so that the community at large can be made aware of IUCD insertion outside of menses.

\section{ACKNOWLEDGEMENTS}

Family Health International (FHI) provided support for this study with funds from United States Agency for International Development. The authors wish to thank Ms. Susan Chen for data management and the staff of the University of Nairobi's research team for data collection and data entry activities. Additionally, we would like to thank the staff and clients of the Kenyatta National Hospital Family Planning Clinic and the Riruta Nairobi City Commission Health Centre for their support and participation. We thank Ms. Linda Ochieng for secretarial services and Mrs. Maureen Kuyoh for constructive comments on the manuscript.

\section{REFERENCES}

1. Hatcher R.A., Trussell J., Stewart F., Cates W. Jr., Stewart G.K., Guest F. and Kowai D. Contraceptive Technology, Ardent Media Inc. New York 1998; 21:522-524.

2. Sinei S.K., Schulz K.F., Lamptey P.R., Grimes D.A., Mati J.K., Rosenthel S.M. and et al.Preventing IUCD-related pelvic infection: the efficacy of prophylactic doxycycline at insertion. Brit. J Obstet. Gynaecol. 1990; 97:412-9.

3. The Kenya health and demographic health survey 1993. Central Bureau of Statistics, Macro International Inc. 4:41-2

4. Qualitative assessment of IUCD service delivery in Kenya. Information Sheet. Fam. Hlth. Int. 1997.

5. White M.K., Ory H.W., Rooks J.B. and Rochat R.W. Intrauterine device termination rates and menstrual cycle day of insertion. Obstet. Gynec. 1979; 55:220-224.

6. Thiery M. Timing of IUCD insertion. Intrauterine Contraception: Advances and future prospects, edited by Zatuchni G., Goldsmith A. and Sciarra J., Philadelphia, Harper and Row. 1985:365-74.

7. Stanback J., Qureshi Z., Sekadde-Kigondu C., Gonzalez B. and Nutley T.A. Checklist for ruling pregnancy among family planning clients in primary care. Lancet 1999; 354:566.

8. Sinei S.K., Morrison C.S., Sekadde-Kigondu C., Allen M. and Kokonya D. Complications of use of intrauterine devices among HIV-1 infected women. Lancet 1998; 351:1238-1241.

9. Reproductive health/family planning policy guidelines and standards for service providers. Government of Kenya 1997; 41-2.

10. Guidelines for clinical procedures in family planning. A reference for trainers, 2nd edition. Intrah 1992; 7:137-168.

11. Hager W.D., Eschenbach D.A., Spence M.R. and Sweet R.L. Criteria for diagnosis and grading of salpingitis. Obstet. Gynaecol. 1983; 61:113-114.

12. Elderman D.A., Zipper J., Rivera M. and Medel M. Timing of IUCD Insertion. Contraception 1979; 19:449-454.

13. IUCDs: Guidelines for informed decision-making and use. Center for Disease Control (CDC), Atlanta, Georgia 1987.

14. Stanback J. Qureshi Z.P., Nutley T., and Gitonga J. Menstruation tally sheet study: East Afr. Med. J. 1999; 76:124-26. 